\title{
COMUNICACIÓN
}

\section{Percepción pública de la existencia y utilidad del Bosque Demostrativo en San Ramón, Costa Rica}

\author{
Flor Elizabeth Araya \& Marta Araúz Almengor \\ Biología, Sede de Occidente, Universidad de Costa Rica, marauzi.arauz@gmail.com \\ Recibido 28-II-2016 • Corregido 20-IV-2016 • Aceptado 24-V-2016
}

\begin{abstract}
Public perception of the existence and usefulness of demonstration forest in San Ramón, Costa Rica. The University of Costa Rica set a small patch of remnant forest in the San Ramon region to preserve the Humid Premontane forest. We surveyed the knowledge and appreciation that the inhabitants of the region have about the forest. We applied two surveys to 237 individuals aged between 8 and 70 years. Most people know the location but few visit or consider the forest beneficial. We recommend improving this aspect when managing the forest.
\end{abstract}

Key words: symbolism, memory, forest conservation, Demonstration Forest.
RESUMEN: La Universidad de Costa Rica estableció un pequeño parche de bosque remanente en la región de San Ramón para preservar el bosque húmedo premontano. Indagamos el conocimiento y apreciación que los habitantes de la región tienen sobre el bosque. Aplicamos encuestas a 237 individuos entre los 8 y 70 años. La mayoría conocen la ubicación del bosques pero pocos lo usan o lo consideran beneficioso. Recomendamos mejorar este aspecto en la gestión del bosque.

Palabras clave: simbolización, memoria, conservación de bosques, Bosque Demostrativo.
Rokeach (1973) afirmó que tenemos dos tipos de valores, terminales e instrumentales, los valores terminales expresan un objetivo en la vida aunque para conseguir ese objetivo existen otros valores relacionados a nuestra vida diaria, por ejemplo, el bien común. Esto es importante en la ética, la cual busca el respeto adecuado por la vida que se preocupe por el bienestar humano desde el ambiente: la ética ambiental va más allá de la cultura Rolston (1991). La ética ambiental tiene que evaluar la naturaleza, tanto la silvestre como la naturaleza que esta mezclada con la cultura.

Los valores que tienen las personas hacia el ambiente se ha complementado en los contenidos del programa de ciencias del Ministerio de Educación Pública de Costa Rica con talleres en la materia ciencias haciendo de sus contenidos interactivos y vivenciales y la transversalidad hacia la conservación del ambiente para la población escolar beneficiaria. La Sede de Occidente de la Universidad de Costa Rica destinó un pequeño parche de bosque remanente que representa lo que fue el bosque original en la comunidad de San Ramón y cantones aledaños, en el cual se conservan especies nativas arbóreas propias de la zona de vida del Bosque Premontano Húmedo. Ofrece una opción educativa y de recreación a los educadores, estudiantes y pobladores de la Región de Occidente de manera que puedan conocer más de cerca las interacciones de los seres vivos representativos de la zona de vida del Bosque Premontano Húmedo y la problemática ambiental (Araúz, 2011).

Se ha incluido la interdisciplinariedad por parte de los estudiantes participantes en trabajo comunal que van a las comunidades a transmitir conocimientos y buscan acciones concretas para la ejecución de proyectos por parte de sus pobladores. Esto incluye la programación de un mantenimiento continuo y sostenible que ha permitido la visitación de grupos familiares, institucionales y de personas individualmente; como respuesta a esa visitación se han organizado talleres que han permitido la divulgación del papel del Bosque Demostrativo y otros aspectos de la conservación de los recursos naturales de la Región de Occidente (Araúz, 2011).

Desde 2005 se abrió el Bosque Demostrativo al público con los llamados "Sábados familiares", los cuales consistieron en brindar un espacio a los núcleos familiares 
y población en general para mostrar la zona de vida del Bosque Premontano Húmedo. La atención a los visitantes la dan los estudiantes con actividades previamente programadas, como: recorridos guiados por los senderos, talleres, explicaciones de los carteles de una exhibición móvil, presentación de videos, cursos, y prácticas demostrativas en el bosque y laboratorio de biología y química (Araúz, 2008).

Bertoni y López (2010) estiman que al hacer evaluaciones sobre la percepción de las personas para una determinada área protegida (reservas, refugios, parques nacionales y otras categorías), los resultados permiten establecer una percepción personal del medio ambiente en función de los valores sociales, ecológicos e intrínsecos, para estimar el grado de satisfacción con el lugar que conocen pudiéndose caracterizar las actitudes ambientales de la población aledaña al bosque y así establecer la potencial conciencia ambiental y conductas pro-ambientales de la población. Aquí estudiamos la percepción personal sobre la existencia y utilidad del Bosque Demostrativo de la Sede de Occidente para estimar las actitudes ambientales por parte de algunos pobladores del cantón de San Ramón.

Se realizó una encuesta con una muestra compuesta de 237 personas de la población ramonense, se seleccionaron al azar. En primer lugar, las personas encuestadas se ubicaron en lugares públicos. Esta encuesta fue aplicada por los estudiantes del curso de Seminario de Realidad Nacional: Salud y Medio Ambiente II, año 2008. Las tres entrevistas incompletas o confusas se eliminaron. Las percepciones relacionadas con el ambiente se definieron como la forma en que cada individuo aprecia y valora su entorno y aportan elementos que potencialmente pueden contribuir a la conservación (Arizpe, Paz \& Velázquez, 1993; Padilla \& Luna, 2003).

La muestra se compuso de $51 \%$ de mujeres. La distribución por grupos de edad fue bastante heterogénea (8 a 70 años). El trabajo de campo se realizó en octubre del 2008 que comprendió tres distritos cercanos (Alfaro (San Pedro), San Isidro y San Ramón centro) a menos de 5 km del Bosque Demostrativo, aproximadamente; el mayor número de personas entrevistadas fueron del centro de San Ramón y San Pedro.

Para el análisis de los cuestionarios se realizaron los siguientes pasos:

Digitalización de datos sobre percepción del Bosque: Se pasaron a formato de Excel un total de 237 encuestas de 30 preguntas cada una, en este proceso se elaboró una base de datos en la que las preguntas de respuesta múltiple se tomaron como preguntas individuales, haciéndose un total de 38 preguntas por encuesta.

Análisis de datos para resultados: Se hizo un análisis descriptivo de los resultados utilizando porcentajes y montando los datos en gráficos y cuadros.

Descripción de la población: La población estudiada correspondió a personas residentes en San Ramón principalmente centro, San Isidro y San Pedro, entre 8 y 70 años con una media de 32 años y una población total encuestada de 237 individuos (Cuadro 1).

Conductas pro ambiental: La encuesta reflejó que cerca de cuatro de cada 10 personas de la población tienen hábitos positivos en cuanto a la conservación del medio ambiente (Figura 1).

Percepción: Según la opinión de las personas encuestadas San Ramón tiene un clima fresco a intermedio (Figura 2).

Funcionalidad del Bosque: Entre las funciones del bosque, las que más sobresale son: las raíces amarran la tierra con un 12\%, además, 37 de cada 100 personas opinan que el bosque cumple por lo menos una de las funciones citadas en la Figura 3.

CUADRO 1

Descripción de la población en cuanto a sexo y escolaridad.

\begin{tabular}{lcc} 
& Femenino (\%) $\mathbf{5 1 , 9 0}$ & Masculino (\%) $\mathbf{4 8 , 1 0}$ \\
Estudios superiores completos & 5,06 & 5,91 \\
Estudios superiores incompletos & 11,81 & 12,66 \\
Primaria completa & 5,91 & 9,28 \\
Primaria incompleta & 5,06 & 2,53 \\
Secundaria completa & 12,24 & 8,44 \\
Secundaria incompleta & 11,81 & 9,28 \\
\hline
\end{tabular}




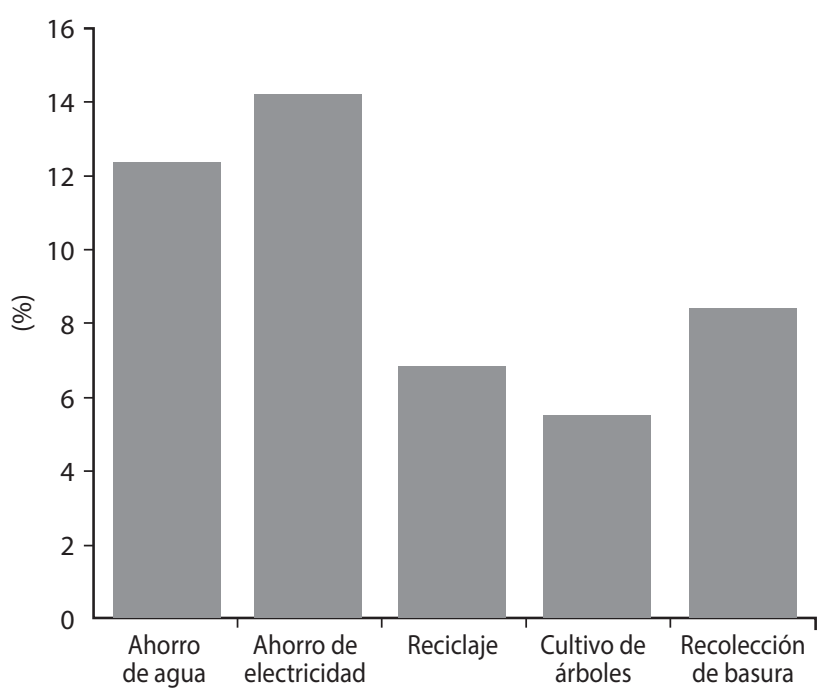

Fig. 1. Principales conductas pro ambiental.

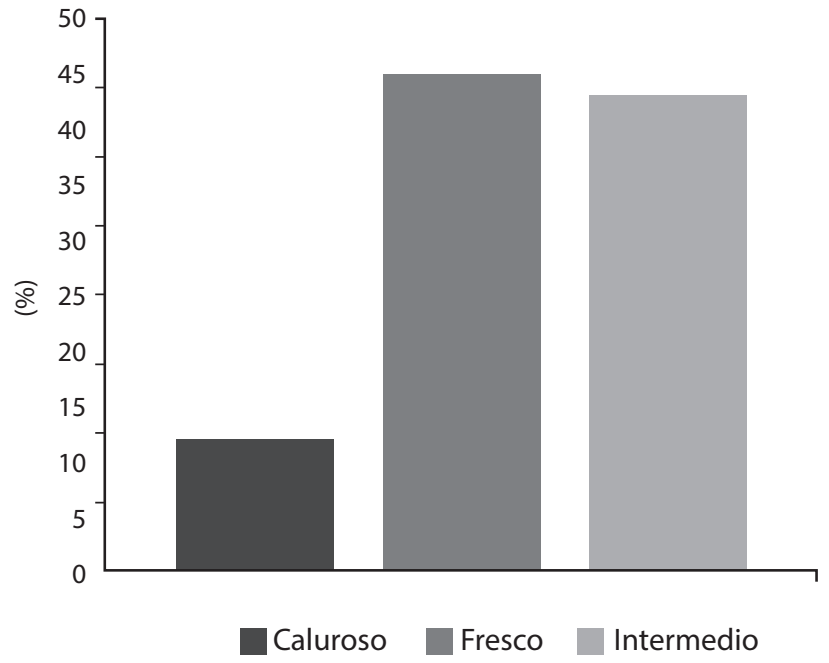

Fig. 2. Percepciones del clima.

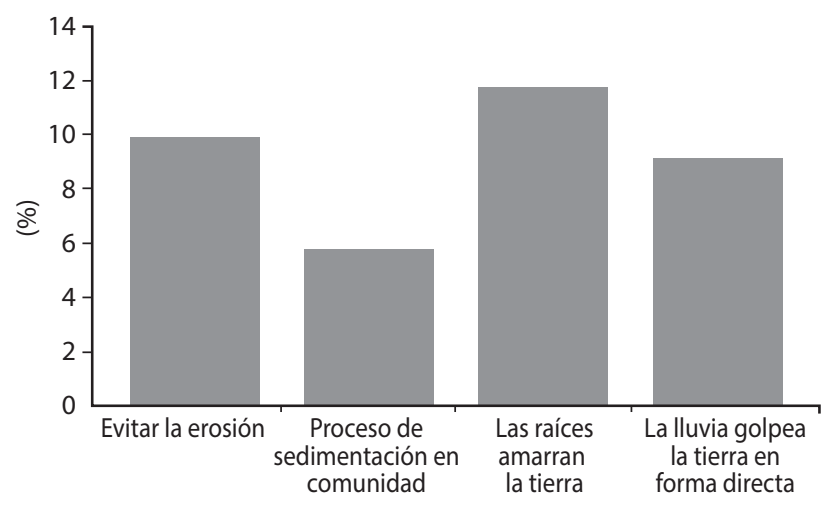

Fig. 3. Principales funciones que cumple el Bosque.

Percepción en el ámbito del Bosque Demostrativo: Sobresalen los datos de que solo 49 de cada 100 personas han hecho uso del Bosque y sin embargo 75 de cada 100 muestran interés en el mismo después de la aplicación de la encuesta. Esto indica que 26 personas más de cada 100 estarían dispuestas a hacer uso o apoyar la iniciativa del Bosque Demostrativo. (Cuadro 2)

Los estudios en percepción de la utilidad y existencia del Bosque Demostrativo mostraron diferentes tipos de beneficios que tienen los pobladores como lo son la regulación de la humedad y temperatura, así como evitar el desgate del suelo por medio de la flora existente en el mismo. Esto es similar a otros resultados (Arizpe, 1993; Araúz 2008, 2011).

Las principales conductas pro ambientales de los ramonenses son el ahorro del agua y la electricidad, estos están ligados al uso eficiente de los recursos en especial los que tienen implicaciones económicas directas y tienen buen potencial de sostenibilidad (Rolston, 1991, Bertoni \& López, 2010; Padilla \& Luna, 2003).

CUADRO 2

Opiniones sobre la utilidad del Bosque Demostrativo de la Sede de Occidente (Bosquecito)

\begin{tabular}{lc} 
& Opiniones \\
El bosquecito incide en la cantidad de humedad & Si (\%) \\
Las actividades recreativas en el bosquecito son de utilidad & 75 \\
Hace usted uso de éstas & 65 \\
El Bosquecito con sus componentes y actividades es importante para el desarrollo de la comunidad & 59 \\
El presente cuestionario ha logrado despertar en usted un interés acerca del bosquecito & 76 \\
\hline
\end{tabular}


La principal preocupación es el desarrollo urbanístico en la percepción del cambio de clima en el cantón, refleja una problemática en relación el agotamiento de recursos naturales, donde no se proponen ni ejecutan alternativas, lo cual puede ser un problema común en comunidades semejantes de la América Latina (Bertoni \& López, 2010).

Los habitantes conocen la ubicación del Bosque Demostrativo según nuestra muestra, pocos lo usan aunque lo consideran beneficioso al tener regulación directa sobre la temperatura y la humedad, aspectos importantes que influyen en disminuir los efectos negativos originados por el cambio climático, principalmente en la pérdida de la biodiversidad y del recurso hídrico. Pacheco, Malizia y Cayuela (2010) encontraron que los bosques premontanos de Argentina y Bolivia son susceptibles a cambios de gran escala por el cambio climático, más de la mitad de este ecosistema se perdería debido al aumento de la temperatura. Por lo tanto, se deben continuar los esfuerzos que hace la Universidad de Costa Rica desde hace 33 años en la conservación de la zona de vida del Bosque Premontano Húmedo que se encuentra en el Bosque Demostrativo de la Sede de Occidente; siendo importante las futuras acciones del manejo del bosque que se realicen conjuntamente con otras instituciones.

\section{REFERENCIAS}

Araúz Almengor, M. L. (2008). Informe Final de Labores Trabajo Comunal Universitario "Bosque Demostrativo de la Sede de Occidente". Coordinación de Acción Social, Sede de Occidente, Universidad de Costa Rica, San Ramón, Alajuela, Costa Rica. 28 pp.

Araúz Almengor, M. L. (2011). Informe Final de Labores Trabajo Comunal Universitario "Bosque Demostrativo de la Sede de Occidente". Coordinación de Acción Social, Sede de Occidente, Universidad de Costa Rica, San Ramón, Alajuela, Costa Rica. 35 pp. .

Arizpe, L., Paz, F \& Velázquez, M. (1993). Cultura y cambio global: percepciones sociales sobre la deforestación en la selva lacandona. México: Centro Regional de Investigaciones Multidisciplinarias-Porrúa. $12 \mathrm{pp}$.

Bertoni, M. \& Lápez, M.J. (2010). Perspectivas socioambientales: valores y actitudes hacia la conservación de Biosfera "Parque Atlántico Mar Chiquita". Estud. Perspect. Tur., 19(5), 835-849.

Pacheco, S., Malizia, L. R. and Cayuela, L. (2010). Effects of climate change on subtropical forests of South America. Tropical Conservation Science Vol. 3 (4): 423-437.

Padilla, L.S. \& Luna, A.M. (2003). “Percepción y conocimiento ambiental en la Costa de Quintana Roo: una caracterización a través de encuestas", Investigaciones Geográficas, $52,99-116$.

Rolston, H. (1991). Environmental Ethics: Values in and Duties to the Natural World. In Bormann \& Kellert Ecology, Economics, Ethics: The Broken circle. Yale university Press. $216 \mathrm{p}$.

Rokeach, M. (1973). The nature of human values. Impreso New York U.S.A. p. 341-354. 